\title{
Belphégor
}

\section{Étude des couvertures de la série des Fantômas dessinées par Gino Starace entre 1911 et 1913}

\section{Annabel Audureau}

\section{(2) OpenEdition}

Journals

Édition électronique

URL : http://journals.openedition.org/belphegor/110

DOI : 10.4000/belphegor.110

ISSN : 1499-7185

Éditeur

LPCM

\section{Référence électronique}

Annabel Audureau, «Étude des couvertures de la série des Fantômas dessinées par Gino Starace entre 1911 et 1913 », Belphégor [En ligne], 11-1 | 2013, mis en ligne le 12 novembre 2013, consulté le 20 juin 2020. URL : http://journals.openedition.org/belphegor/110 ; DOI : https://doi.org/10.4000/belphegor. 110

Ce document a été généré automatiquement le 20 juin 2020.

\section{c) $(1) \&$}

Belphégor est mis à disposition selon les termes de la Licence Creative Commons Attribution - Pas d'Utilisation Commerciale - Pas de Modification 4.0 International. 


\title{
Étude des couvertures de la série des Fantômas dessinées par Gino Starace entre 1911 et $1913^{1}$
}

\author{
Annabel Audureau
}

1 Héritier des bandits masqués qui règnent sur la littérature populaire depuis le siècle précédent, Fantômas est un homme sans visage, un insaisissable bandit dont les aventures, imaginées pour la maison d'édition Fayard par Pierre Souvestre et Marcel Allain à partir de 1911, ont enchanté des milliers de lecteurs de la Belle Époque. Rebelle à la volonté de classification de son temps, résistant au " bertillonnage ${ }^{2}$ » ambiant, le criminel se définit aussi comme un homme aux cent visages, as du postiche, dont les multiples apparences constituent autant de paravents empêchant son identification par les forces de l'ordre incarnées principalement par le commissaire Juve.

2 En utilisant l'image, son image, pour arriver à ses fins, Fantômas apparaît comme une métaphore des couvertures des livres populaires de son époque: un miroir aux alouettes, une apparence souvent trompeuse qui vise à séduire le lecteur potentiel. Car le rôle essentiel de la première de couverture de la littérature de masse qui se développe dans le courant du dix-neuvième siècle est de stimuler l'achat, de susciter le désir, de retenir l'attention du passant. En devenant un objet de consommation, le livre a besoin d'un "emballage ", comme le fait remarquer Charles Grivel dans son article consacré à la couverture du roman illustrés. C'est l'illustrateur Gino Starace qui va mettre en scène les méfaits fantômassiens dans la collection du «Livre populaire » entre 1911 et 1913, créant ainsi un véritable cinéma imprimé propre à marquer les esprits et à séduire les chalands comme le souligne, dès 1907, Van Dooren dans sa préface à L'Anthologie illustrée des Poètes et des Prosateurs de France et de Belgique :

Ils se pressent aux vitrines des librairies ou des marchands de journaux et là, les yeux écarquillés, le cou tendu, ils béent d'admiration devant la grossière imagerie des illustrés à un sou, ils se bousculent pour mieux voir, jusqu'au plus petit détail, les pages sanguinaires et imbéciles, où hurlent les couleurs les plus criardes - il faut bien n'est-ce pas, aguicher le passant ${ }^{4}$ ! 
La fonction publicitaire de la première de couverture des romans populaires semble donc évidente et, par là-même, la signification de l'image devient, selon les termes de Roland Barthes, intentionnelle ${ }^{5}$, les signes constitutifs y fonctionnant à plein, choisis en vue du déchiffrage le plus explicite.

4 Concernant la lecture des premières de couverture de ce type de romans, doit-on alors parler de facilitation ou de manipulation? Selon Daniel Couégnas ${ }^{6}$, l'image donne à voir, elle est un judas ouvert sur le texte à venir, alors que Charles Grivel ${ }^{7}$ relève le paradoxe lexical qu'induit le terme de "couverture » dans sa fonction intrinsèque de découverte... Si la couverture illustrée apparaît comme un objet accessible, cela ne la rend pas simple pour autant. Vitrine offrant son visage le plus séduisant, elle n'en demeure pas moins un support de créativité et un sujet d'étude complexe.

\section{Une fausse lisibilité ?}

5 En couverture de la série des Fantômas, on trouve une structure invariante composée d'une illustration dessinée par Gino Starace et un paratexte. De manière récurrente, ce dernier précise que "Chaque volume forme un récit complet», soulignant ainsi la particularité formelle de la série par opposition au feuilleton, très en vogue à l'époque. Il s'agit bien entendu d'un argument d'ordre économique qui assure au futur lecteur un achat rentable en lui proposant une lecture cohérente en une acquisition unique et non fragmentée comme cela est le cas pour le feuilleton. Tout sera fait cependant pour stimuler l'achat d'autres volumes : page introductrice indiquant les titres des volumes qui précèdent et qui suivent, notes en bas de page rappelant les épisodes précédents... Sur chaque première est également indiqué le prix, modique : le somme promotionnelle de 35 centimes pour le premier volume puis 65 centimes. L'argumentaire économique s'affiche donc sans complexe sur la devanture de la littérature de masse, dite aussi « de consommation ", qui se doit pour prospérer d'adopter les lois du marché.

On remarque ensuite un autre groupe paratextuel qui a pour fonction, plus implicite, de faciliter la démarche d'identification de l'objet par le lecteur. Le processus de réification propre à la littérature populaire engendre une mise en valeur du "quoi » sur le « qui », de la production et de sa réalité concrète (l'objet-livre), sur l'auteur. La couverture illustrée va même plus loin en privilégiant le contenant par rapport au contenu. La typographie utilisée sur les couvertures de la série met ainsi davantage en vedette le personnage de Fantômas que ses géniteurs (différence de la taille des caractères et des couleurs). Le nom des auteurs n'est clairement pas le déclencheur de l'achat du livre. Ce que l'on achète, ce n'est pas un récit de Pierre Souvestre et Marcel Allain, mais une nouvelle aventure de l'Insaisissable. Et le contrat passé avec Fayard stipulait d'ailleurs que les auteurs pouvaient être remplacés. Plus le bandit masqué fait recette, plus le levier identificatoire fonctionne. En achetant un volume, on entre dans le groupe des lecteurs de Fantômas et c'est toujours cette identification que réactivent les artistes d'avant-garde, à l'instar de Guillaume Apollinaire qui fonde le Club des Amis de Fantômas. Cependant, en faisant entrer par effraction le plus populaire des bandits dans la sphère artistique. Ce dernier joue sur le décalage et l'originalité de cette muse improbable en inversant proprement l'enjeu commercial initial. Le consensus est brisé et devient subversif voire révolutionnaire avec les surréalistes ${ }^{8}$ ! 
Couverture du premier volume publié par Arthème Fayard et affiche du premier film de Louis Feuillade

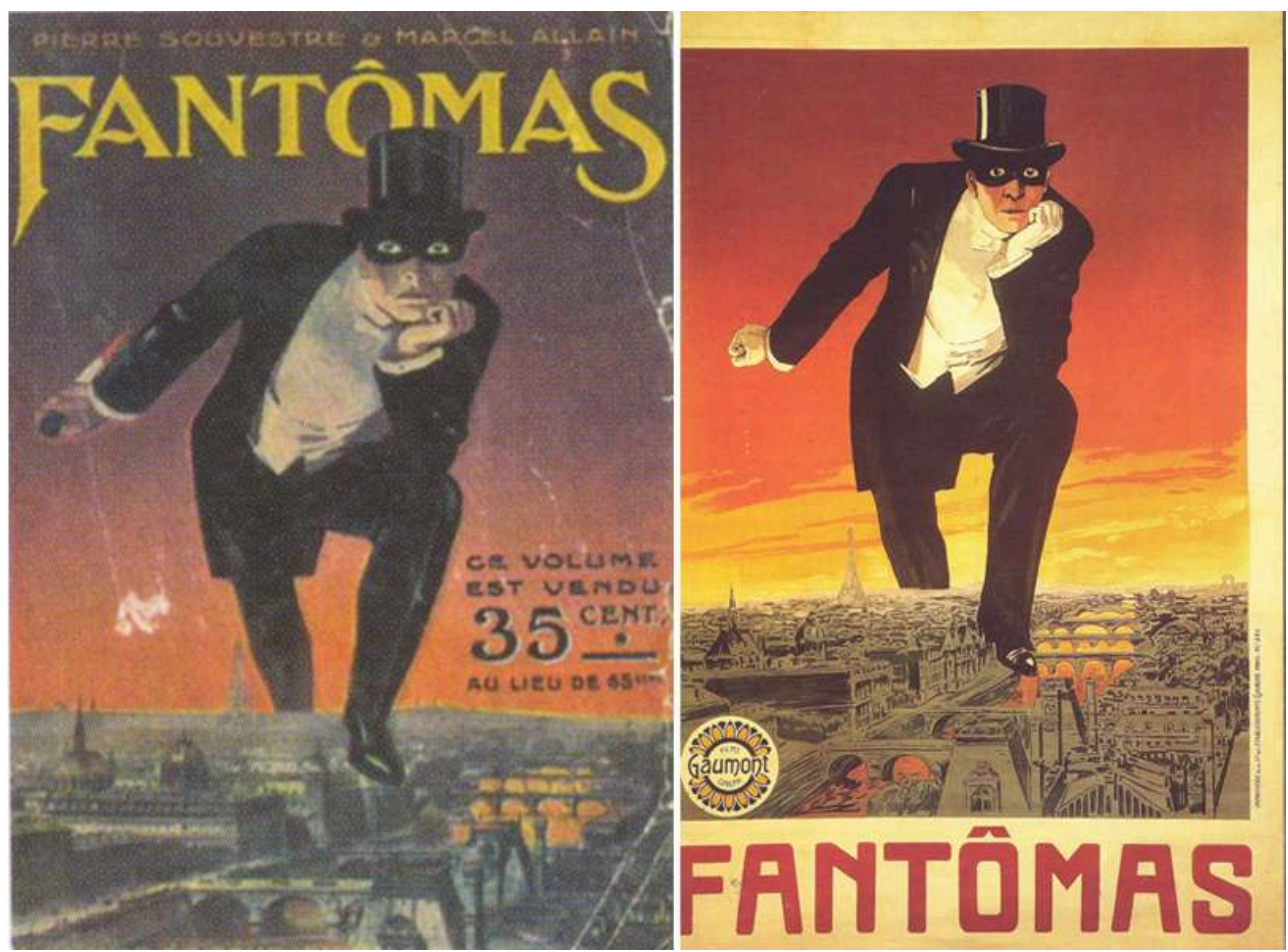

Fayard, 1911 et Gaumont, 1913 
$7 \mathrm{Au}$ bas de la couverture, on trouve des indications sur la maison d'édition et la collection, le paratexte venant ainsi encadrer les dessins dont le rôle est également de renvoyer à un "déjà-vu», "déjà-lu ». La genèse de la couverture inaugurale de Fantômas rend particulièrement compte de cette recherche d'adhésion immédiate. Ainsi, la plus connue des couvertures de la série, qui inspira des peintres surréalistes comme René Magritte $^{9}$ et sera largement médiatisée par le biais des affiches des adaptations cinématographiques de Louis Feuillade en $1913^{10}$, représente-t-elle Fantômas sous l'apparence stéréotypée du bandit masqué, très en vogue à la Belle Époque, période durant laquelle la société française tend à thésauriser et donc à craindre particulièrement le vol ${ }^{11}$... En tenue de dandy, costume noir, chemise blanche et haut-de-forme, Fantômas y apparaît masqué d'un loup noir. Brandissant un poignard ensanglanté dans sa main droite, il enjambe les ponts de Paris dans un ciel rougi par le coucher du soleil... Gino Starace ${ }^{12}$ reprend ici à son compte des codes visuels efficaces, et ne laisse nulle place à la polysémie, la multiplicité vagabonde de la lecture de l'image. S'il y a poésie, comme en témoigne l'engouement des peintres surréalistes, notamment René Magritte, pour cette couverture, elle est involontaire. Il s'agit ici de toucher sa cible en plein cœur. L'image révèle donc des signes immédiatement décodables par le public. Le loup, associé à la tenue de dandy, rappelle d'autres élégants bandits masqués tels Rocambole ou, plus tard, Arsène Lupin. La ville de Paris, facilement identifiable par des éléments d'architectures symboliques comme la Tour Eiffel ou les ponts surplombant la Seine, est aperçue en plongée et représentée de nuit, rappelant les décors d'autres séries à succès illustrées par Starace comme Les Mystères de Paris d'Eugène Sue ou Paris Mystérieux de Ponson du Terrail. La redondance visuelle permet la constitution d'un ensemble de codes, une " grammaire visuelle simplifiée ${ }^{13}$ " qui renvoie le lecteur, même médiocrement instruit, à un système de références connu.

Cette couverture-ouverture vise donc à présenter le personnage principal d'une nouvelle série, tout en l'inscrivant dans un univers rassurant pour le lecteur. De fait, tous les éléments constitutifs du récit sont présents. Le poignard, recouvert de sang, annonce le caractère sanguinaire de Fantômas, alors que la capitale et plus particulièrement le Palais de justice qu'il semble piétiner avec désinvolture, seront son terrain de jeu privilégié. Rien de neuf cependant, Fantômas appartient à la famille notoire, et déjà rentable, du bandit masqué. Héros de recyclage? La première de couverture se base en tout cas sur des recettes connues et emprunte d'ailleurs sa mise en scène visuelle à un autre. Ainsi, à la recherche d'une idée d'illustration, les auteurs de Fantômas fouillent-t-ils dans les cartons de la maison Fayard pour exhumer une affiche publicitaire sur laquelle un géant vante les mérites des pilules "Pink » censées redonner couleurs et vigueur, dont il arrose généreusement la ville de Paris qu'il enjambe à l'instar de Fantômas sur la couverture s'en inspirant. Double ironie cependant que de remplacer des pilules prometteuses de vigueur contre une arme blanche et de démarrer la carrière du plus grand des imposteurs par un quasi-plagiat ! Pourtant, si l'Insaisissable se laisse circonscrire dans la famille des bandits masqués, il entend les dépasser tous par sa cruauté et sa suprématie que symbolisent son arme dégoulinante de sang et sa posture hiératique qui, même si elle se démarque du reste des productions de Gino Starace par une fixité insolite pour celui qui passe le plus clair de son temps à échapper aux forces de l'ordre, traduit un règne sans partage possible de la terreur et du crime dont on trouve un écho dans l'incipit du roman : 
- Fantômas! - Vous dites ? - Je dis... Fantômas - Cela signifie quoi ? - Rien... et tout ! - Pourtant qu'est-ce que c'est ? - Personne... mais cependant quelqu'un! Enfin que fait ce quelqu'un ? - Il fait peur ! ! !14 s'amusent d'ailleurs de cette intertextualité : «Juve, vous en êtes encore à Rocambole et Rocambole est mort...", se moque le jeune Fandor, ce à quoi Juve répond: « $\mathrm{Si}$ Rocambole est mort, Fantômas existe ${ }^{15}$.» Il existe, et il a acquis une suprématie sur ses prédécesseurs grâce à l'évolution des nouvelles technologies de ce début du vingtième siècle, notamment la vitesse des déplacements accrue par le biais des chemins de fer qui datent déjà du siècle précédent (voir par exemple la couverture dessinée par Starace pour illustrer La Fille de Fantômas, huitième roman de la série, sur laquelle une locomotive lancée à vive allure, à laquelle est attachée Hélène, donne littéralement l'impression de sortir du cadre), mais aussi, et surtout, grâce aux automobiles dont les auteurs étaient par ailleurs passionnés et grands connaisseurs ${ }^{16}$. George Clémenceau fonde, à cette même époque, les brigades policières en automobile, immortalisées sous le nom de "Brigades du Tigre ». "La police est beaucoup mieux faite de nos jours qu'autrefois ${ }^{17} \ldots$ » souligne d'ailleurs l'abbé Sicot lors d'une soirée chez la marquise de Langrune, dans le premier opus des aventures de Fantômas faisant écho à cette évolution de la société :

Sans doute, reconn(aît) le président, mais son rôle est plus difficile aussi, que jamais! Les bandits de haute envergure ont, pour exécuter leurs forfaits, beaucoup de moyens à leur disposition; la science si favorable aux progrès modernes, peut à l'occasion, hélas! devenir un véritable collaborateur des criminels! Par conséquent, les chances s'égalisent de part et d'autre ${ }^{18}$ ! »

10 Mais si la couverture inaugurale de la série dessinée par Starace se révèle être un empilement de masques, assurant ainsi une lisibilité orientée, et visant à inscrire le lecteur dans un univers de signes qui fait sens, la suite de sa production témoigne également d'un art de la narration et d'une créativité qui, même si elle est mise au service de la commercialisation d'un produit, existe cependant.

\section{Une vraie narrativité...}

11 À partir de 1905, Starace se consacre entièrement à la collection du "Livre populaire " lancée par Arthème Fayard. C'est ainsi qu'il collabore en 1911 avec Pierre Souvestre et Marcel Allain. Son travail d'illustration participe du succès de la série et de l'inscription de Fantômas dans l'imaginaire collectif. Expressivité outrancière, dynamisme violent, tension extrême vers un hors cadre, définissent les trente-et-une couvertures (la série initiale en comptera trente-deux) que Starace va réaliser pour la série des Fantômas. On peut les apparenter à l'art pompier, par leur représentation d'un sujet (un passage du roman ou ayant un rapport avec le récit), la mise en scène stéréotypée des passions (qu'on lit dans la gestuelle emphatique ou les expressions outrancières des visages) mais également par l'utilisation d'une narration chronologique dans une image figée qui donne pourtant l'illusion du mouvement qui se dégage notamment de la structure des dessins de Gino Starace et du mouvement des corps croqués dans des positions de tension extrême à la limite du possible anatomique : ainsi Hélène, sur la couverture de La Fille de Fantômas semble-t-elle sur le point de se désarticuler, engagée dans le même mouvement que le train auquel elle est accrochée. 


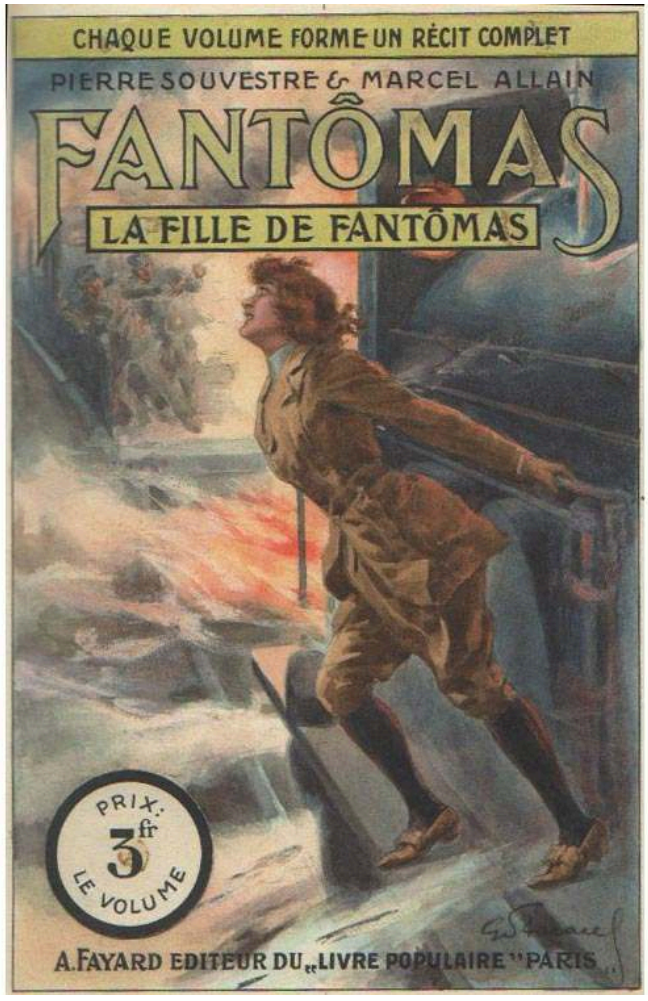

«Le Livre populaire », Arthème Fayard, 1911

12 Afin de toucher le lectorat le plus large, l'illustrateur puise dans des domaines de représentation populaires comme celui du mélodrame, du dessin de presse (notamment celui des faits divers que l'on retrouve par exemple dans L' (Eil de la Police) et du cinématographe. La couverture du tome XX : Les Souliers du mort présente ainsi un gros plan sur un visage en proie à la plus grande terreur. Yeux exorbités, mains crispées sur la bouche, la mise en scène des expressions faciales s'apparente à celle des acteurs de mélodrame, celle que l'on retrouve sur les écrans des cinémas où les mêmes acteurs recyclent un jeu hyperbolique afin de suppléer à l'absence de son des films muets ${ }^{19}$. Même le visage d'un mort conserve une expressivité propre à marquer les esprits comme celui de la baronne de Vibray sur la couverture du Mort qui tue (tome III), ou, plus grand-guignolesque, celui de Ribonard (Le Magistrat cambrioleur, tome XII), un apache à la solde de Fantômas, dont le corps ensanglanté ayant remplacé le battant d'une cloche, semble vouloir sortir du cadre imposé par l'objet-livre, entraînant le spectateur dans son balancement funèbre... Par ailleurs, l'illustration du Policier apache montre en couverture une bande d'apaches dont la représentation se rapproche de l'imagerie véhiculée par la presse de faits divers: coiffés de casquettes à pont, ils revêtent une veste cintrée et un pantalon large. 


\section{Couverture du Policier Apache}

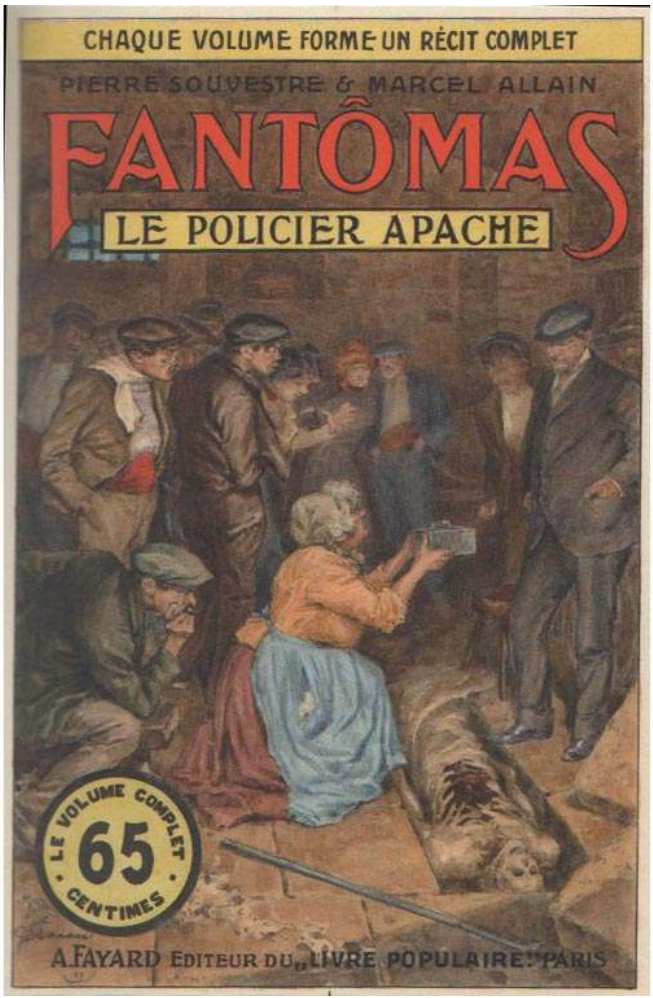

« Le Livre populaire », Arthème Fayard, 1911

13 Cependant, l'utilisation de codes déjà connus ne doit pas occulter une mise en scène graphique complexe du récit illustré. Selon Daniel Couégnas :

(...) L'illustration de couverture doit être une sorte de miroir, ou mieux, de judas, par lequel le futur lecteur jette un coup d'œil pour y apercevoir l'un des temps forts de l'action que le romancier distillera au long des centaines de pages $(. . .)^{20}$.

La maitrise académique du graphisme figuratif rend apparemment indissociables référent et représentation par un effet d'illusion référentielle. Ainsi, la couverture de Juve contre Fantômas reprend-elle un extrait du seizième chapitre. Afin d'échapper à l'incendie qui ravage les Halles de Bercy, Juve et Fandor se sont glissés dans un tonneau vide :

Le tonneau ouvert, rempli d'escarbilles, de fumées, de tisons, roula, roula... Cependant qu'à l'intérieur Juve et Fandor, contusionnés, ballottés, brûlés... étaient incapables désormais de résister et de lutter contre l'effroyable rapidité de l'extraordinaire véhicule qu'ils avaient adopté pour se sauver ${ }^{21}$. 


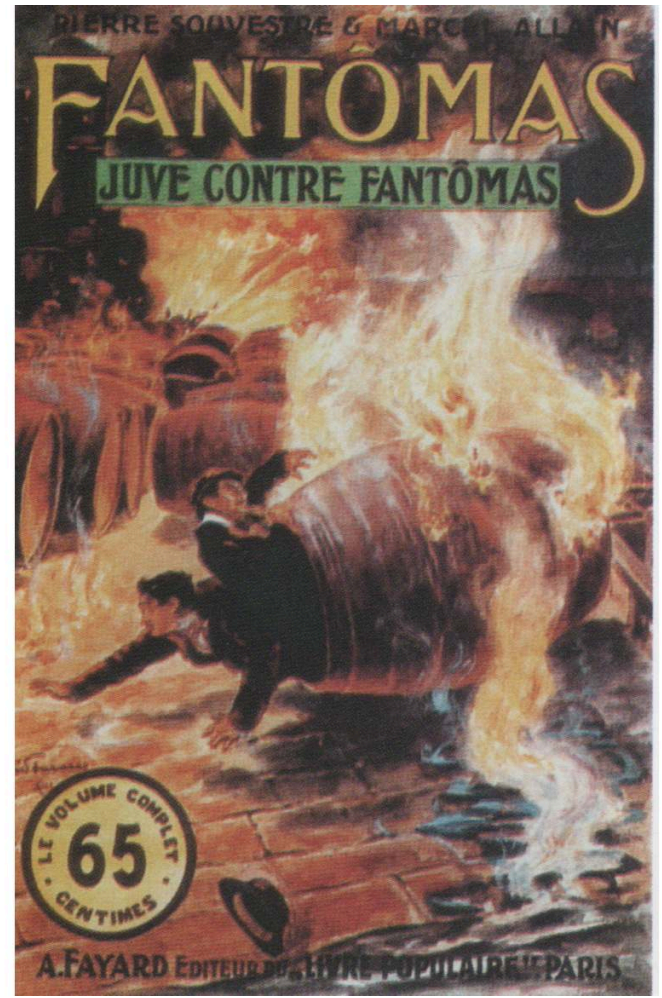

« Le Livre populaire », Arthème Fayard, 1911

15 L'illustration de Starace reprend fidèlement la scène sur le plan du référent. On retrouve le même cadre spatial, les mêmes personnages et une action identique. Mais le signifié est également traduit. Les nombreuses figures d'exagération comme les utilisations emphatiques du vocabulaire: "effroyable", "extraordinaire», ou les énumérations : «[...] rempli d'escarbilles, de fumées, de tisons [...]» participent d'une écriture de l'excès que l'illustrateur adapte au médium visuel. Les corps contorsionnés des deux hommes, ainsi que les mains tendues, concourent à la théâtralisation de la gestuelle et traduisent l'intensité de l'action dramatique alors que les visages expriment l'émotion des personnages de façon expressive. Favorisée par les évolutions techniques, mise en vedette par le cinématographe, l'image acquiert, en effet, un pouvoir de séduction de plus en plus grand. Moment choisi de la narration, la couverture appelle un hors-cadre, celui même que doit vendre l'illustration. Les dessins de Gino Starace, en donnant l'illusion du mouvement, insistent sur la dynamique même $\mathrm{du}$ récit et cette technique est particulièrement efficace pour rendre compte de la poursuite interminable entre Fantômas, le criminel, et Juve, le policier. Roman de la quête plutôt que de l'enquête, Fantômas, l'homme comme le récit, est ennemi du fixisme et l'illustrateur de la maison Fayard possède le don de rendre la vie à ce que fige le crayon, d'attirer l'œil du passant vers un hors-cadre prometteur, d'ouvrir la couverture. 


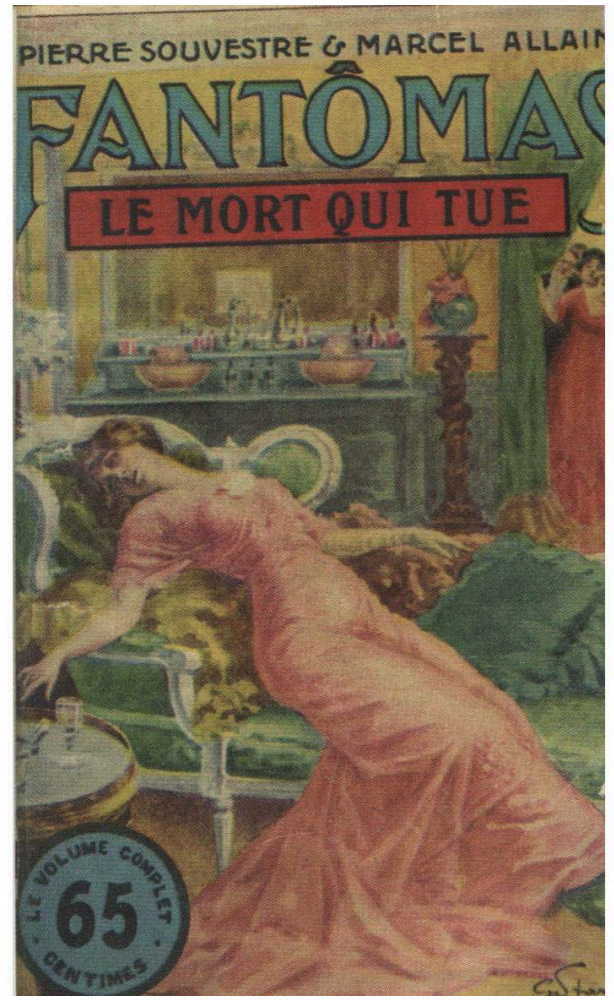

«Le Livre populaire », Arthème Fayard, 1911

Pour cela Gino Starace privilégie des compositions en diagonale qui permettent au spectateur de s'intégrer à l'action en le plaçant au même niveau que le point de vue des personnages de la scène. La couverture du Mort qui tue $e^{22}$ présente ainsi une image décomposable en trois plans. Au premier plan, un guéridon placé dans l'angle gauche du cadre, débute une diagonale qui se prolonge dans le bras inerte de la baronne de Vibray dont le corps inanimé repose sur un sofa au deuxième plan. Son bras gauche, étendu de l'autre côté de son corps, souligne la présence d'un troisième plan, presque une coulisse à cette scène théatralisée. Un rideau, ainsi que des témoins, renforcent cette impression d'être le témoin privilégié du spectacle, plus proche encore que les protagonistes de la scène dont le déroulement à venir se trouve au-delà du point de fuite, derrière la couverture. L'image construite par Gino Starace assure ainsi le spectacle propre à sa nature visuelle, retient l'attention, mais sans voler la vedette au texte, qu'elle encourage, par sa composition, à consulter afin d'en savoir plus. L'illustration de L'Agent secret ${ }^{23}$ fonctionne sur le même schéma. Au premier plan, Bobinette dérobe, sous les yeux du lecteur potentiel, une carte d'état major, secret qu'ignore son amant, demeuré à l'arrière-plan, derrière le même rideau entr'ouvert que l'on trouvait sur la première de couverture du Mort qui tue. Le point de fuite de l'image suit le regard de ce personnage vers l'arrière-plan où l'on aperçoit le capitaine Brocq. La perspective tend à accentuer l'illusion de profondeur et de secret. L'arrièreplan devient alors métaphorique d'un ailleurs textuel que le personnage qui lit met en abyme, alors que le premier plan établit une connivence avec le lecteur en en faisant le témoin du vol de la carte. Dans le même temps, l'illustrateur place le lecteur dans une position privilégiée tout en lui promettant la révélation d'autres secrets. L'agent secret, c'est le lecteur. 


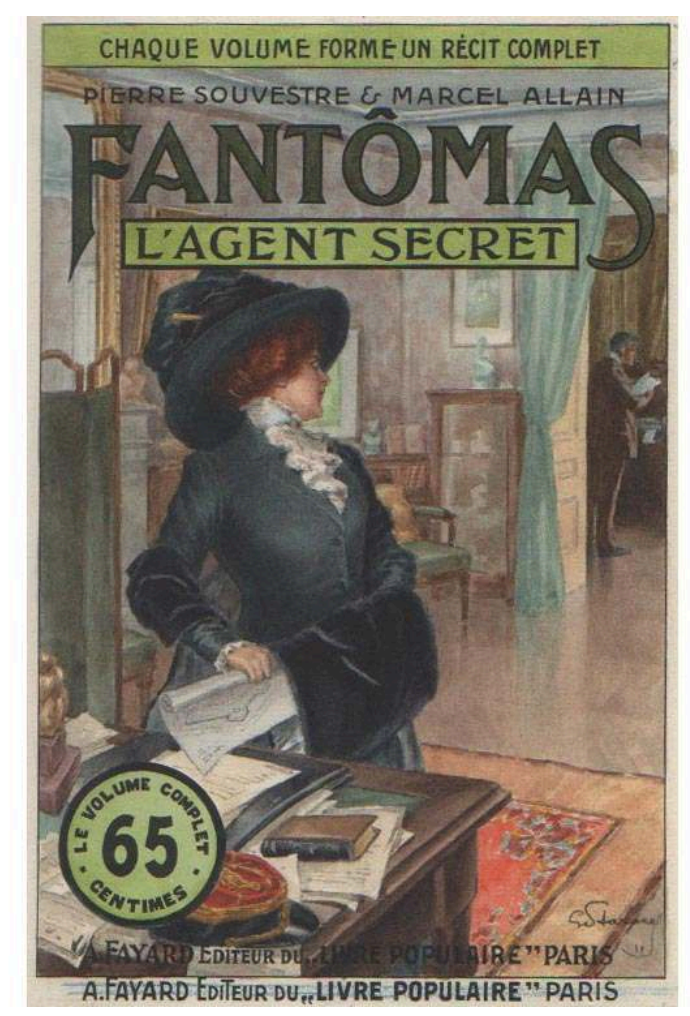

«Le Livre populaire », Arthème Fayard, 1911

17 Afin de «saisir " une scène censée se poursuivre hors du cadre de la couverture, les dessins de Gino Starace donnent l'illusion du mouvement, détrônant encore pour quelques années le jeune cinématographe mais s'en inspirant également. Par ses compositions dynamiques, l'illustrateur entend sortir du cadre, suggérer un ailleurs convoqué au sein même de la fixité du dessin. Certaines de ses couvertures donnent d'ailleurs l'impression d'un instantané, qui se rapproche davantage d'un photogramme saisissant l'action dans son déroulement que du cliché photographique de l'époque auquel il manque cette illusion du mouvement. C'est le cas d'Hélène, sur la couverture de la Fille de Fantômas (volume VIII) ou de Ribonard attaché à sa cloche pour Le Magistrat cambrioleur (volume XII). Quant aux illustrations de L'évadée de Saint Lazare ${ }^{24}$ ou de L'assassin de lady Beltham ${ }^{25}$, elles représentent chacune une scène d'accident saisie dans son déroulement, se rapprochant également des techniques du dessin de presse, en particulier celle du fait divers, domaine où la photographie n'a pas encore la suprématie qu'on lui connaît aujourd'hui. En amorçant une action: celle du corps tendu d'Hélène qui tente d'échapper à un train fou, ou de l'omnibus de Montmartre qui semble foncer sur le spectateur, Gino Starace transforme en atout le caractère statique de l'art graphique car il crée un arrêt au sein même de la narration visuelle. Il ménage ainsi un suspens propre à éveiller la curiosité du lecteur comme lorsqu'il campe la silhouette noire de Fantômas qui surgit sous le lit de sa victime endormie sur la couverture du volume XVI (La disparition de Fandor $\left.{ }^{26}\right)$, invitant le lecteur à anticiper le geste d'agression... 

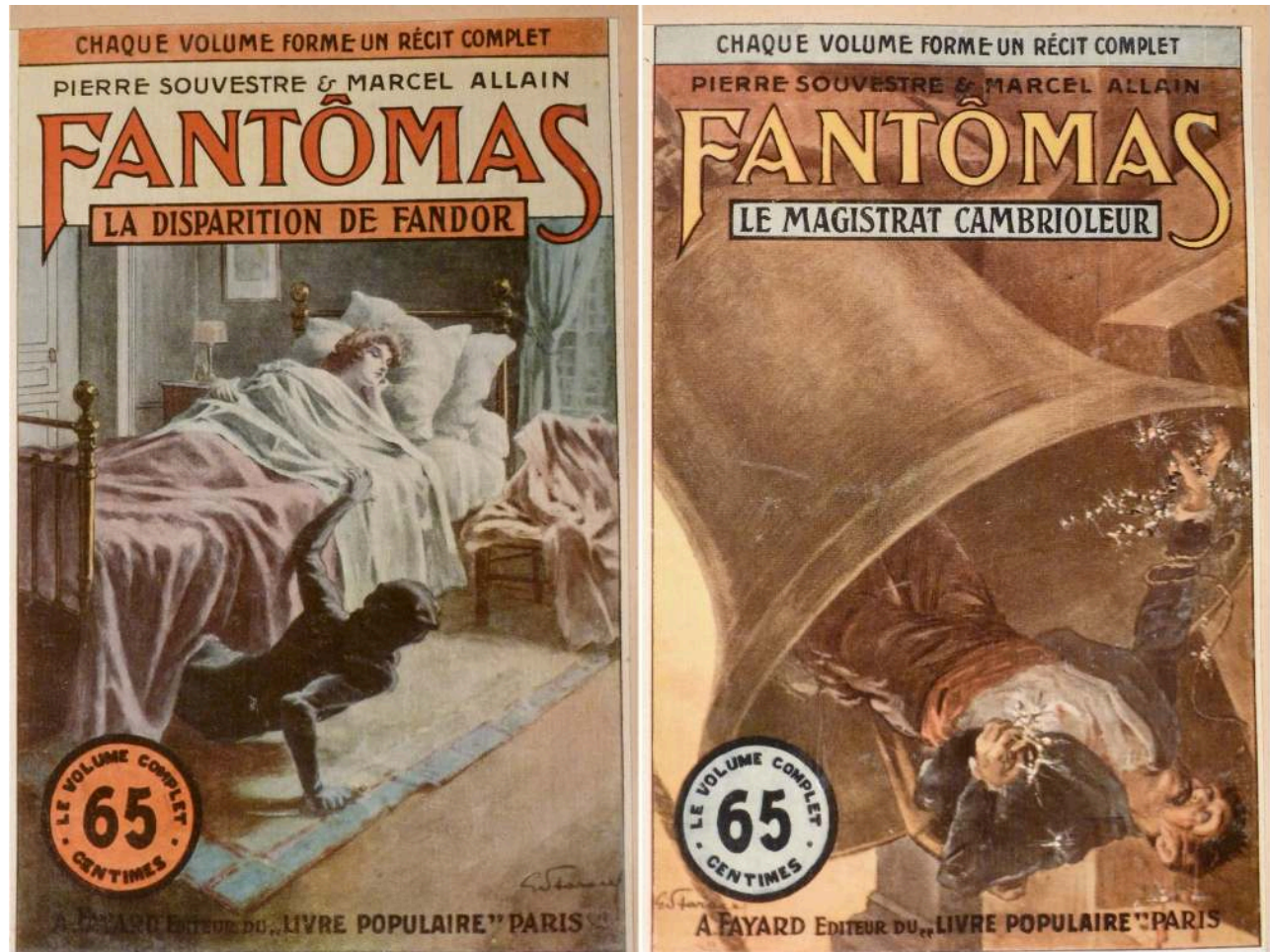

« Le Livre Populaire », Arthème Fayard, 1912 chaland en affichant, à la devanture des kiosques, le spectacle chamarré des crimes de Fantômas. Séduire par la peur, par la fascination pour la mort et la violence, quitte à user de l'imagerie grand-guignolesque en illustrant d'une main coupée et sanguinolente, le volume $\mathrm{X}$ au titre éponyme ... Eros et Thanatos ne sont jamais bien loin. Mais la couverture doit aussi assurer une lisibilité immédiate plus rationnelle : les indications précisant l'appartenance à une série aux volumes complets, les prix et même les promotions, font également partie de l'arsenal argumentatif.

Gino Starace dessine à l'apogée de l'art de l'illustration; les visées commerciales de ses productions ont guidé la créativité de l'illustrateur de la maison Fayard; elles ne l'ont pas empêché de marquer durablement les esprits et de participer à la construction de Fantômas en mythe moderne. L'image a ainsi concouru à la construction d'une représentation symbolique de Fantômas. Le loup, la tenue de dandy et le poignard ensanglanté deviennent les attributs d'une figure qui s'ancre dans la mémoire collective, dont l'impact se trouve redoublé par la reproduction liée à la commercialisation de la littérature de masse. La couverture sera reprise plus tard par les peintres surréalistes comme René Magritte ${ }^{27}$, lui-même inspiré par le médium cinématographique (les adaptations de Fantômas au cinéma par Louis Feuillade). Ainsi, l'image commerciale forge-t-elle un imaginaire propre à créer de nouveaux mythes dont Fantômas sera. Porteur de la modernité de son temps, il connaîtra ainsi un parcours artistique fécond tout au long du vingtième siècle croisant à la fois les champs d'expression d'avant-garde et les productions populaires tant en littérature, qu'en peinture ou au cinéma. 
René Magritte, Retour de flamme

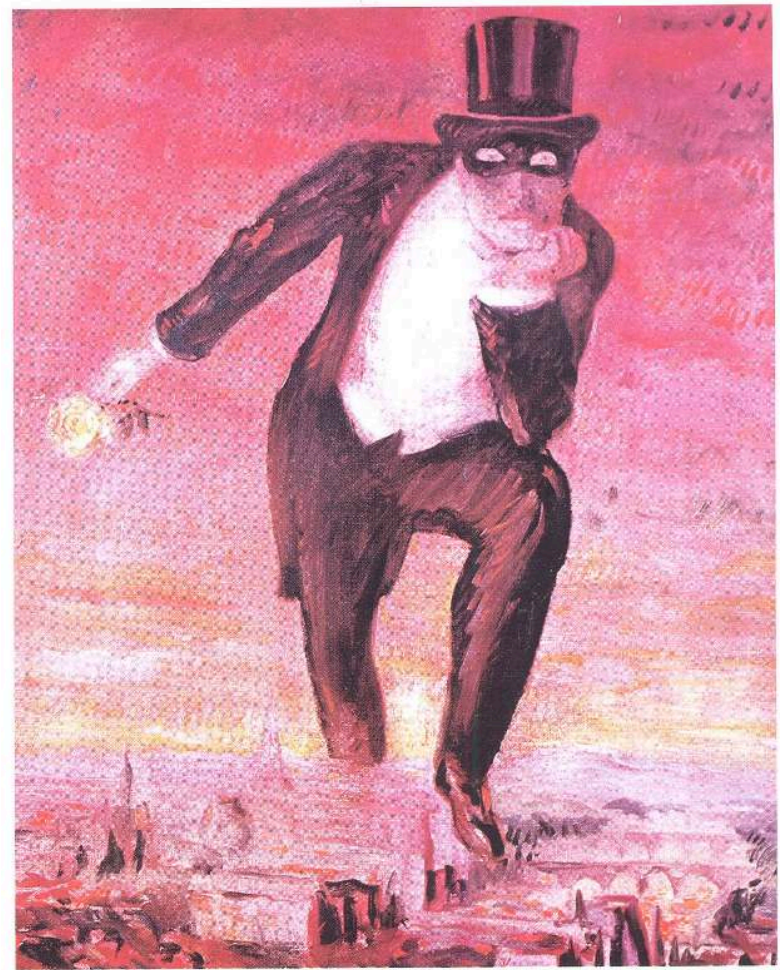

D.R.

\section{BIBLIOGRAPHIE}

Corpus de 32 volumes de la série des Fantômas parus chez Arthème Fayard dans la collection du «Livre populaire » et illustrés par Gino Starace :

1. Fantômas, 10 février 1911.

2. Juve contre Fantômas, $1^{\mathrm{er}}$ mars 1911.

3. Le Mort qui tue, 20 avril 1911.

4. L'Agent secret, 20 mai 1911.

5. Un Roi prisonnier de Fantômas, 20 juin 1911.

6. Le Policier apache, 20 juillet 1911.

7. Le Pendu de Londres, 20 août 1911.

8. La Fille de Fantômas, 20 septembre 1911.

9. Le Fiacre de nuit, 20 octobre 1911.

10. La Main coupée, 20 novembre 1911. 
11. L'Arrestation de Fantômas, 17 décembre 1911.

12. Le Magistrat cambrioleur, 17 janvier 1912.

13. La Livrée du crime, 20 février 1912.

14. La Mort de Juve, 11 mars 1912.

15. L'Évadée de Saint-Lazare, 20 avril 1912.

16. La Disparition de Fandor, 20 mai 1912.

17. Le Mariage de Fantômas, 6 juin 1912.

18. L'Assassin de lady Beltham, 20 juillet 1912.

19. La Guêpe rouge, 20 août 1912.

20. Les Souliers du mort, 20 septembre 1912.

21. Le Train perdu, 20 octobre 1912.

22. Les Amours d'un prince, 20 novembre 1912.

23. Le Bouquet tragique, 20 décembre 1912.

24. Le Jockey masqué, 20 janvier 1913.

25. Le Cercueil vide, 20 février 1913.

26. Le Faiseur de reine, 20 mars 1913.

27. Le Cadavre géant, 20 avril 1913.

28. Le Voleur d'or, 20 mai 1913.

29. La Série rouge, 20 juin 1913.

30. L'Hôtel du crime, 20 juillet 1913.

31. La Cravate de chanvre, 20 août 1913.

32. La Fin de Fantômas, 20 septembre 1913.

Alfu, Gino Starace, l'illustrateur de Fantômas, Amiens, Encrage, 1988, non paginé.

Audureau Annabel, Fantômas : un mythe moderne au croisement des arts, Rennes, PUR,

«Interférences », 2010.

Couégnas Daniel, «Les couvertures illustrées du roman populaire à travers l'œuvre de Gino Starace : lisibilité, emphase, narrativité » in Fictions, Énigmes, Images, Limoges, PULIM, 2001.

Courtes Joseph, Du lisible au visible, Bruxelles, De Boeck Université, 1995.

Grivel Charles, « De la couverture illustrée du roman populaire », in Production(s) du populaire, actes réunis par Jacques Migozzi et Philippe Le Guern, Limoges, PULIM, 2004.

\section{NOTES}

1. Le corpus de notre étude comprend les trente-deux premiers volumes de la série par chez Arthème Fayard entre 1911 et 1913 dans la collection du « Livre Populaire ».

2. Alphonse Bertillon fonde en 1870 le premier laboratoire de police d'identification criminelle et invente l'anthropométrie judiciaire, appelée « système Bertillon » ou « bertillonnage ». 
3. Charles Grivel, «De la couverture du roman illustré » in Production du populaire, Limoges, PULIM, 2004, p. 281 à 299.

4. J. Van Dooren, Préface à l'édition de 1907 de son Anthologie Illustrée des Poètes et des Prosateurs de France et de Belgique, Belgique, Desoer, 1925.

5. Selon Roland Barthes, les signifiés de la publicité, représentés par les caractéristiques du produit, doivent être assimilés chez le spectateur de la meilleure manière possible : «si l'image contient des signes, on est donc certain qu'en publicité ces signes sont pleins, formés en vue de la meilleure lecture : l'image publicitaire est franche ou du moins empathique ", Roland Barthes, «Rhétorique de l'image » in Communication 4, Paris, Seuil, Ecole pratique des Hautes Etudes, 1964, p. 40.

6. Couégnas Daniel, «Les couvertures illustrées du roman populaire à travers l'œuvre de Gino Starace : lisibilité, emphase, narrativité » in Fictions, Énigmes, Images, Limoges, PULIM, 2001, p. 60.

7. Charles Grivel, « De la production du roman illustré », op. cit., p. 281.

8. Fantômas a, en effet, inspiré de nombreux artistes d'avant-garde: Guillaume Apollinaire, Blaise Cendrars, Max Jacob mais aussi Louis Aragon, André Breton, Ernst Moerman ainsi que des peintres comme Yves Tanguy, René Magritte, voir pour une étude plus approfondie du phénomène : Fantômas : un mythe moderne au croisement des arts, Rennes, Presses Universitaires de Rennes, «Interférences », 2010, ouvrage de l'auteur du présent article.

9. René Magritte, Le Retour de flamme, huile sur toile, $65 \times 60 \mathrm{~cm}$, collection particulière, 1943.

10. L'affiche du premier film de Louis Feuillade, intitulé : Fantômas, à l'ombre de la guillotine, offre cependant une version édulcorée, le poignard ensanglanté de l'insaisissable brandit, ayant été censuré.

11. Il est intéressant de souligner que le terme de "cambriolage » entre dans le dictionnaire Larousse à la Belle Époque.

12. Cette première couverture, qui n'est pas signée, est d'un style très différent de celles de Starace. Il est très probable qu'elle ne soit pas de lui.

13. Terme emprunté à Daniel Couégnas, «Les couvertures illustrées du roman populaire à travers l'œuvre de Gino Starace : lisibilité, emphase, narrativité », op. cit., p. 61.

14. Pierre Souvestre et Marcel Allain, Fantômas, Paris, Arthème Fayard, «Le Livre populaire ", 1911, p. 61.

15. Pierre Souvestre et Marcel Allain, Juve contre Fantômas, Paris, Arthème Fayard, «Le Livre populaire ", 1911, p. 124.

16. Leur premier roman, Le Rour, qui annonce certaines des thématiques de Fantômas, est publié dans le journal L'Auto et a pour but de vanter la qualité des pneumatiques Ducable! Pierre Souvestre a également rédigé un dictionnaire des termes techniques de l'automobile en 1901 ainsi qu'une Histoire de l'Automobile publiée chez Dunod. Il ouvre par ailleurs un garage automobile et devient propriétaire du magazine Le Poids Lourd en 1909.

17. Pierre Souvestre et Marcel Allain, Fantômas, op. cit., p. 9.

18. Ibid., p. 14.

19. Les adaptations de Louis Feuillade témoignent cependant d'une recherche de naturel dans le jeu des acteurs de la part du metteur en scène, tout autant que d'une volonté d'échapper à la tutelle du théâtre en favorisant les tournages en extérieur et les scènes d'actions, nombreuses dans les aventures de Fantômas.

20. Daniel Couégnas, «Les couvertures illustrées du roman populaire à travers l'œuvre de Gino Starace : lisibilité, emphase, narrativité ", op. cit. p. 63-64.

21. Pierre Souvestre

Souvestre

et Marcel Allain

Allain 
, Juve contre Fantômas, Paris, Fayard, « Le Livre populaire », 1911, p. 61.

22. Pierre Souvestre et Marcel Allain, Le Mort qui tue, Paris, Arthème Fayard, «Le Livre populaire ", 1911.

23. Pierre Souvestre et Marcel Allain, L'Agent secret, Paris, Arthème Fayard, « Le Livre populaire ", 1911.

24. Pierre Souvestre et Marcel Allain, L'Évadée de Saint Lazare, Paris, Arthème Fayard, «Le Livre populaire ", 1912.

25. Pierre Souvestre et Marcel Allain, L'Assassin de lady Beltham, Paris, Arthème Fayard, « Le Livre populaire ", 1912.

26. Pierre Souvestre et Marcel Allain, La disparition de Fandor, Paris, Arthème Fayard, « Le Livre populaire ", 1912.

27. René Magritte, Le Retour de flamme, huile sur toile, $65 \times 60 \mathrm{~cm}$, collection particulière, 1943.

\section{RÉSUMÉS}

Les couvertures que Gino Starace a dessinées pour la collection du «Livre populaire » chez Arthème Fayard ont pleinement participé aux succès des romans publiés dans cette collection. C'est le cas de la série des Fantômas imaginée par Pierre Souvestre et Marcel Allain à partir de 1911. Fonctionnant comme un cinéma imprimé, ces illustrations ouvrent une fenêtre colorée et expressive sur les aventures de papier bon marché de l'insaisissable bandit masqué et ont pour but de séduire le lecteur potentiel en s'affichant à la devanture des kiosques de la Belle Époque. Elles offrent ainsi des objets de sémiologie complexes et éclairants sur la fonction de la première de couverture dans la paralittérature.

\section{INDEX}

Mots-clés : culture populaire, littérature populaire, avant-garde, cinéma, illustration, Fantômas

\section{AUTEUR}

\section{ANNABEL AUDUREAU}

Annabel Audureau est docteur en littérature comparée et enseigne l'expression et communication à l'IUT de La Rochelle. Elle est aussi chargée de cours à l'université de La Rochelle en littérature française et comparée. Spécialisée dans le domaine de la littérature populaire et des avant-gardes, elle a écrit un livre sur le mythe de Fantômas publié aux éditions des Presses Universitaires de Rennes en 2010 et s'intéresse également à l'analyse du cinéma (organisation et participation à des colloques ainsi que cours sur le cinéma et publications). 